\title{
Determinantes do Requerimento de Colateral em Empréstimos Empresariais*
}

\author{
Jaimilton Carvalho ${ }^{\dagger}$, José Angelo Divino ${ }^{\ddagger}$, Jaime Orrillo ${ }^{\S}$
}

Conteúdo: 1. Introdução; 2. Modelo Econométrico; 3. Estimação Logit para Dados em Painel; 4. Resultados; 5. Conclusão; A. Apêndice A - Regressor endógeno; B. Apêndice B - Estimação Logit com dados em painel.

Palavras-chave: Colateral; Empréstimos comerciais; Setor financeiro; Dados em Painel.

Códigos JEL: G21; D43; E32.

O objetivo deste artigo é avaliar, empiricamente, determinantes do requerimento de colateral exigido pelo credor do setor empresarial formal da economia brasileira, no horizonte de curto prazo. Os resultados empíricos indicam que a probabilidade de vinculação de colateral em empréstimos empresariais é maior entre devedores com menor qualidade de crédito e entre devedores com maior qualidade de crédito não observada pelo credor. Adicionalmente, maior competição e rivalidade no mercado de crédito elevam aquela probabilidade. Tais evidências se mostram em conformidade com a literatura recente, no sentido de que requerimentos de colateral podem ser distintos entre diferentes mercados de crédito.

The goal of this paper is to evaluate the determinants of collateral requirement by the lender in the formal entrepreneurial sector of the Brazilian economy in the short-run horizon. The results indicate that the probability of using collateral is higher among borrowers with lower credit quality and borrowers who fulfill past financial promises. In addition, higher competition and rivalry in the credit market increase the probability of collateral utilization in short-run loans. Such evidence corroborates recent findings of the literature in the sense that the determinants of collateral requirement in financial loans might be distinct among different credit markets.

\footnotetext{
* Os autores agradecem ao Editor, um parecerista anônimo e aos participantes no XXXIX Encontro Nacional de Economia da ANPEC pelos valiosos comentários e sugestões. Os autores agradecem ao convênio ANPEC/BNDES pelo financiamento deste estudo. José Angelo Divino e Jaime Orrillo agradecem ao CNPq pelo apoio financeiro. Todos os erros remanescentes são de responsabilidade exclusiva dos autores. As opiniões expressas neste trabalho são independentes da afiliação institucional dos autores.

† Banco do Brasil e Universidade Católica de Brasília,E-mail: jaimilton@hotmail . com

‡Universidade Católica de Brasília,E-mail: jangelo@pos.ucb.br

§Universidade Católica de Brasília, Programa de Pós-Graduação em Economia. SGAN 916, office A-116, Asa Norte, Brasilia - DF, Zip: 70.790-160. Brasil. Fone: +55(61)3448-7192. Fax: +55(61)3347-4797. E-mail: orrillo@pos.ucb.br
} 


\section{INTRODUÇÃO}

O objetivo deste artigo é avaliar, empiricamente, quais são os determinantes do requerimento de colateral pelo setor bancário em um segmento específico do mercado de crédito, o empresarial formal, levando-se em consideração tanto as características dos tomadores quanto da conjuntura macroeconômica do país.

Atualmente, condições domésticas e externas de mercados financeiros têm mostrado que diversos fatores podem influenciar a exigência de colateral por parte dos bancos em operações de crédito para empresas. Nesse sentido, observa-se que aquele requerimento pode ser afetado não apenas por características dos tomadores, mas também por diversos outros aspectos do mercado financeiro, incluindo condições micro e macroeconômicas. Na literatura, esta questão vem sendo muito investigada.

Do ponto de vista da teoria econômica que considera a existência de informação assimétrica no relacionamento de empréstimos entre credores e devedores, observa-se o desenvolvimento de uma extensa literatura, teórica e empírica. Esta foca em diversos temas, incluindo a modelagem de mecanismos de filtro ou de sinalização que evidenciem ou minimizem efeitos provenientes do problema de informação assimétrica em mercados financeiros. Dentre estes, são referenciados, por exemplo, a utilização de capital próprio como um sinalizador de qualidade de crédito em projetos de investimentos empresariais, de acordo com trabalho seminal de Leland e Pyle (1977), e o uso de colateral como mecanismo de incentivos e auto-seleção em mercados de crédito com seleção adversa, conforme Bester (1985) e Chan e Kanatas (1985).

Especificamente quanto ao uso de colateral, estudos anteriores também apontam diversas variáveis que podem afetar aquela exigência do credor quando da concessão de empréstimos comerciais. Dentre elas, a existência de uma relação direta entre risco de crédito observado de devedores e requerimento de colateral em operações de empréstimos, como destacam Boot et alii (1991). Em determinados contextos, duration ${ }^{1}$ pode contribuir para construção de confiança e compartilhamento de risco e redução daquele requerimento (Boot e Thakor, 1994). Por outro lado, um maior tempo de relacionamento bancário pode estar associado a uma maior probabilidade de vinculação de colateral, caso duration esteja associado ao efeito hold-up, ${ }^{2}$ conforme Greenbaum et alii (1989) e Farinha e Santos (2002), por exemplo. Além disso, se as firmas dependem de crédito bancário, mercados competitivos podem contribuir para redução do tempo de relacionamento, ou não, se bancos decidem investir em fidelização de clientes [Petersen e Rajan $(1995,1994)$ e Boot e Thakor (2000)]. ${ }^{3}$

Estudos mais recentes acrescentam que mercados de crédito parecem funcionar de forma diferente entre devedores com distintos níveis de experiência e para empréstimos bancários comerciais com maturidades distintas (Jiménez et alii, 2006).O efeito conjunto de garantias fisicas e pessoais pode impactar positivamente na relação entre emprestador e tomador [Voordeckers e Steijvers (2006) e Ono e Uesugi (2009)]. De acordo com a teoria do colateral lender-based, proposta por Inderest e Mueller (2007), inovações tecnológicas e proximidade da oferta de crédito podem afetar as taxas de juros e o requerimento de colateral. Contudo, considerando-se também a distância organizacional na oferta de crédito, Jiménez et alii (2009) observam que, sob determinadas características de devedores, o uso do colateral é superior em empréstimos concedidos pelos credores locais do que naqueles concedidos por credores mais distantes. Uma survey atual desta literatura pode ser obtida em Berger et alii (2011), no qual os autores

\footnotetext{
${ }^{1}$ Nesta literatura o termo duration é comumente utilizado para representar o tempo de relacionamento financeiro existente entre credor e devedor.

${ }^{2}$ Situação em que as duas partes, credor e tomador, poderiam agir cooperativamente, mas se recusam a fazer isso para evitar aumentar o poder de barganha da outra parte e, com isso, reduzir seus próprios benefícios.

${ }^{3}$ Especificamente para o caso brasileiro, há estudos que também consideram efeitos de risco de crédito, duratione competição bancária, como Nakane (2002), De Araújo e Jorge-Neto (2007) e Ozawa e Nakane (2009). Diferentemente desses estudos anteriores, porém, aqui se testa diretamente hipóteses relacionadas com o problema de informação assimétrica e exigência de colateral em empréstimos empresariais.
} 
consideram possíveis benefícios provenientes de avanços em mercados de crédito ou de inovações políticas adotadas pelo credor para minimização de efeitos de informação assimétrica e uso de colateral em contratos de crédito.

A inspiração desse estudo é Jiménez et alii (2006), que notaram a possível distinção entre os determinantes de colateral em empréstimos bancários comerciais com respeito aos prazos pactuados e níveis de experiência de devedores e sugeriram que investigações futuras poderiam focar em segmentos do mercado de crédito. Incorpora, também, sugestão de Steijvers e Voordeckers (2009) para que se possa selecionar a ferramenta econométrica mais adequada à evidência empírica almejada.

Logo, serão analisados os determinantes do requerimento de colateral em empréstimos bancários comerciais para um segmento específico do mercado de crédito brasileiro, o segmento empresarial formal, no horizonte de curto prazo. Para tanto, será utilizada uma base de dados inédita sobre operações de crédito formalizadas em que, além de características de firmas individuais e condições macroeconômicas, serão incluídas informações contratuais, regionais e setoriais. O banco de dados exclusivo está estruturado na forma de painel e cobre cerca de 170 mil empréstimos empresariais no período de dezembro de 2004 até dezembro de 2008. As estimativas são obtidas via aplicação de regressão logística e método dos momentos generalizados (GMM), conforme exigido pela hipótese a ser testada acerca dos determinantes do requerimento de colateral exigidos pelo credor em empréstimos ofertados para pequenos e médios empresários na economia brasileira.

As principais contribuições do artigo referem-se à construção de uma base de dados exclusiva e realização de testes inéditos sobre os determinantes do requerimento de colateral em empréstimos bancários pactuados com empresas formais na economia brasileira. Os resultados obtidos sugerem, em conformidade com a literatura recente, que requerimentos de colateral podem ser distintos entre mercados de crédito e que, sob regime de taxas elevadas de juros, o credor pode escolher exigir um maior valor de colateral com o intuito de minimizar efeitos de problemas de moral hazard. Além disso, a probabilidade de vinculação de colateral em empréstimos comerciais é maior entre aqueles devedores com elevada qualidade de crédito, indicando que o efeito dominante na amostra analisada se refere ao grupo que detém informação privada no momento da contratação do empréstimo.

O artigo está organizado da seguinte forma. A próxima seção apresenta o modelo econométrico utilizado nas estimativas e descreve as hipóteses a serem testadas. A terceira seção discute o modelo logit para dados em painel. A quarta seção apresenta os dados, reporta e analisa os resultados obtidos. Por fim, a quinta seção é dedicada às observações conclusivas.

\section{MODELO ECONOMÉTRICO}

O ponto de partida da análise é o modelo proposto por Jiménez et alii (2006), chamado JSS (2006) para simplificar, que servirá de base para especificação do modelo econométrico e para a definição das hipóteses que serão testadas acerca dos determinantes da exigência de colateral em empréstimos empresariais na economia brasileira. Assim, o modelo econométrico aplicado à estimação da probabilidade de vinculação de colateral por pequenas e médias empresas individuais assume a seguinte forma geral:

$$
\begin{array}{r}
\operatorname{Pr}\left(\text { Collateral }_{j t}=1\right)=F\left(b_{0}+b_{1} \text { Default }_{j t-1}+b_{2} \text { Default }_{j t+1}+b_{3} \text { Duration }_{j t-1}+\right. \\
b_{4} \text { Default }_{j t-1} x \text { Duration }_{j t-1}+b_{5} \text { Herfindahl }_{t}+ \\
\left.b_{6} \text { Herfindahl }_{t} \text { Duration }_{j t-1}+b_{7} \text { ControlVariables }_{j t}+n_{j}\right)
\end{array}
$$

onde $t$ representa o período de tempo medido em anos, $j$ define as unidades empresarias, $n_{j}$ representa efeitos fixos de cada firma individual $j, F\left(\right.$.)é uma função de distribuição logística e, Collateral $_{j} t$ é uma variável dummy que assume valor 1 se há um colateral vinculado à operação de crédito e 0 caso contrário. As demais variáveis são descritas conforme se segue: 
- Default ${ }_{j t-1}$ variável dummy que assume valor $1 \mathrm{se}$, de acordo com a base de dados, no momento do empréstimo o empresário tem pelo menos uma operação de crédito inadimplente em $t-1$, e 0 caso contrário;

- Default ${ }_{j t+1}$ variável dummy que assume valor $1 \mathrm{se}$, de acordo com a base de dados, no momento do empréstimo o empresário não tem registro histórico de inadimplência em $t-1$ mas apresenta pelo menos uma operação inadimplente em $t+1$, e 0 caso contrário;

- Duration $_{j t-1}$ representa o tempo de relacionamento de empréstimos entre a firma e o banco brasileiro, medido em anos, conforme sugerido por JSS (2006).

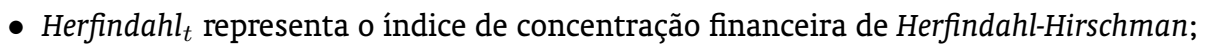

- ControlVariables ${ }_{j} t$, representam variáveis de controle incluídas na regressão com o intuito principal de investigar impactos de condições macroeconômicas sobre o requerimento de colateral em operações de empréstimos empresariais, tais como taxa de inflação, taxa básica de juros da economia e taxa de desemprego. Outras variáveis de controle incluídas na regressão referem-se a características de devedores, tais como idade, porte da firma, natureza jurídica, total de dívidas existentes e utilização de meio eletrônico para efetuar operações bancárias, setor de atuação da firma (comércio, indústria, serviços), unidade da federação e risco geográfico. Este último é medido pela proporção entre empréstimos de baixa qualidade e o total de empréstimos concedido em cada estado brasileiro.

A estimativa por dados em painel justifica-se pela possibilidade de controlar para heterogeneidade específica a cada firma, permitindo que se capturem efeitos de choques individuais que são firmaespecífica, além de choques agregados dependentes do tempo e que afetam todas as firmas indistintamente. Além disso, a modelagem por painel possibilita ampliar consideravelmente o número de observações da amostra e introduz maior variabilidade aos dados. A seleção prévia entre os modelos alternativos, representados por efeitos fixos ou efeitos aleatórios, pode ser realizada por meio teste de Hausman (1978). Note-se que o resultado desse teste gera uma evidência particular, uma vez que a presença de efeitos fixos pode ser interpretada como um prêmio de risco que é devedor-específico.

\subsection{Descrição de Hipóteses}

Com base em JSS (2006), serão testadas as seguintes hipóteses acerca dos determinantes da exigência de colateral em empréstimos empresariais por parte do setor bancário na economia brasileira:

Hipótese 1 (H1): Do ponto de vista do risco observado, a probabilidade de vinculação de colateral em operações de crédito é maior entre devedores com menor qualidade de crédito;

Hipótese 2 (H2): Entre devedores que possuem informação privada a respeito da qualidade de crédito, não factível de observação pelo credor, a probabilidade de usar colateral é maior entre aqueles com maior qualidade de crédito;

Hipótese 3a (H3a): A probabilidade de utilização de colateral diminui com o tempo de relacionamento em empréstimos entre credor e devedor, chamado duração para simplificar, se os benefícios daquele relacionamento dominam. Por outro lado, aquela probabilidade aumenta com a mencionada duração se os custos de hold-up problems associados ao relacionamento de empréstimo dominam;

Hipótese 3b (H3b): Entretanto, se um longo tempo de relacionamento devedor-credor contribui para gerar confiança, então, a duração será negativamente correlacionada com a probabilidade de utilização de colateral para todos os devedores. Por outro lado, se uma longevidade no mencionado 
relacionamento significa que credores tornam-se mais precisos na classificação de tomadores de crédito, então, a probabilidade de vinculação de colateral será independente da duração para aqueles devedores cuja qualidade de crédito é conhecida no momento da concessão do empréstimo;

Hipótese 4a (H4a): A probabilidade de exigência de colateral diminui com a maior concentração do mercado de crédito;

Hipótese 4b (H4b): A redução na probabilidade de vinculação de colateral proveniente de uma longa duração será maior (menor) em mercados mais concentrados se o valor de informação obtida no relacionamento diminui (aumenta) com a competição no mercado de crédito;

Hipótese 5 (H5): $O$ valor de colateral requerido em um contrato de crédito bancário elevará com a taxa de juros livre de risco e diminuirá com o tamanho do empréstimo.

Os resultados teóricos que suportam a formulação das hipóteses anteriores, assim como, o procedimento de teste da equação (1), respectivas variáveis utilizadas como proxie e sinais esperados para os coeficientes, encontram-se detalhados em JSS (2006). Por isso, não serão descritos neste artigo.

Observa-se ainda que hipóteses relacionadas às seguintes variáveis: nível de especialização de credores com respeito à avaliação de risco de crédito de devedores, quantidade de bancos com os quais as empresas se relacionam e distância organizacional, esta última avaliada em Jiménez et al. (2009), não serão testadas nesta avaliação empírica em função de limitações acerca da disponibilidade de informações no banco de dados utilizado na análise. Ainda assim, a riqueza de informações contidas no painel de pequenas e médias empresas permite que se teste diversas hipóteses inéditas a estudos sobre esse mercado na economia brasileira.

Além disso, diferentemente de JSS (2006) e exclusivamente no teste da quinta hipótese acima descrita, será utilizada a seguinte variável dependente:

$$
\text { Fcollateral }_{j t}=\left(1+\text { CollateralValue }_{j t} / \text { ContractValue }_{j t}\right)
$$

onde Fcollateral $_{j t}$ representa um fator discreto de percentuais de colateral vinculados em empréstimos bancários empresariais. Nota-se que para construção deste fator são utilizadas as variáveis CollateralValue $_{j t}$, representando valores de colateral vinculados em contratos de crédito, e ContractValue ${ }_{j t} \neq 0$, representando valores totais de empréstimos tomados pela firma individual. Então, o modelo econométrico para testar (H5) se torna:

$$
\begin{array}{r}
\text { Fcollateral }_{j t}= \\
b_{0}+b_{1} \text { Default }_{j t-1}+b_{2} \text { Default }_{j t+1}+b_{3} \text { Duration }_{j t-1}+b_{4} \text { Default }_{j t-1} \\
\times \text { Duration }_{j t-1}+b_{5} \text { Herfindahl }_{t}+b_{6} \text { Herfindahl }_{t} \times \text { Duration }_{j t-1}+b_{7} \\
\text { ContractValue }_{j t}+b_{8} \text { ontrolVariables }_{j t}+n_{j}+u_{j t}=X_{j t}^{\prime} . b+e_{j t}
\end{array}
$$

em que $e_{j t}=n_{j}+u_{j t}$ é um termo de erro composto por $n_{j}$ e $u_{j t}$, os quais representam choques invariantes ao longo do tempo específicos a cada empresa individual $j$ e choques idiossincráticos variantes no tempo, respectivamente.

É importante observar, na equação (2) anterior, que a variável ContractValue ${ }_{j} t$ pode ser considerada endógena, conforme mostrado no apêndice A.

\section{ESTIMAÇÃO LOGIT PARA DADOS EM PAINEL}

Uma questão central em estimativas com dados de painel é a seleção do modelo a ser utilizado na regressão, representado por efeitos fixos ou aleatórios, porque envolve endogeneidade de regressores 
devido à possibilidade de que $E\left[x_{j t} . n_{j}\right] \neq 0$. Logo, para se proceder com a estimativa deve-se previamente selecionar entre modelos de efeitos fixos ou aleatórios, o que será feito por meio do teste de Hausman (1978), com a hipótese nula: $E\left[x_{j t} . n_{j}\right] \neq 0$. Sob essa hipótese, a estimação por FGLS (Feasible Generalized Least Squares) é consistente e eficiente. Sob a hipótese alternativa, FGLS é inconsistente e o modelo a ser adotado é o de efeitos fixos. O Apêndice B descreve a representação Logit para dados em painel.

Vale observar que, sob endogeneidade de regressores, torna-se necessário o uso de variáveis instrumentais. Neste caso, esta análise utilizará o método dos momentos generalizados, GMM, que permite recuperar a consistência e eficiência dos estimadores, conforme mostram Hansen (1982) e Hamilton (1994). É importante notar que a utilização do GMM requer a verificação de aspectos relacionados com a sobreidentificação de parâmetros e validade do conjunto de instrumentos, o que será feito por meio da aplicação dos testes de Sargan (1958) e Sargan-difference. ${ }^{4}$

Observa-se ainda que a estimação com dados de painel e regressão logística, o cálculo do efeito marginal aproximado de uma variável $x_{j t}$ sobre a probabilidade de resposta $\operatorname{Prob}\left(y_{j t}=1 \mid x\right)$ seguirá Wooldridge (2001).Quanto ao cálculo de semi-elasticidades, a análise acompanhará Jiménez et alii (2006), ou seja, este é obtido por meio da multiplicação do valor do efeito marginal aproximado pelo valor médio de cada variável.

\section{RESULTADOS}

\subsection{Dados}

Esta análise empírica utiliza aproximadamente 170 mil observações mensais de contratos de empréstimos comerciais de curto prazo, no período de dezembro de 2004 a dezembro de 2008 . As variáveis de interesse foram construídas apenas para as empresas que permaneceram na base durante o período proposto para análise, constituindo um painel balanceado.

Tal período é justificado pela disponibilidade de dados e variáveis específicas de contratos de crédito, assim como, pela relativa estabilidade econômica observada na economia brasileira, motivo pelo qual o ano de 2009 não foi incluído na estimativa. Nesse ano, a crise financeira internacional iniciada nos Estados Unidos em 2008 afetou a atividade econômica interna do país, que experimentou uma queda de $0,33 \%$ no produto per capita.

A base de dados inédita é construída a partir de informações públicas e privadas, disponibilizadas em diversas fontes no mencionado período. O tratamento das variáveis "Natureza jurídica" e "porte da firma" segue critérios utilizados pelo Instituto Brasileiro de Geografia e Estatística (IBGE). Os dados macroeconômicos e regionais são obtidos no Ipeadata. Já as informações sobre índice de concentração de mercado de crédito são disponibilizadas pelo Banco Central do Brasil. Por fim, dados contratuais de empréstimos bancários de curto prazo são provenientes de uma base de dados exclusiva e confidencial de um grande banco brasileiro, líder no mercado de empréstimos a pequenas e médias empresas na economia brasileira, motivo pelo qual fonte e dados não podem ser divulgados.

\subsection{Estimações e Análises}

As estimativas dos determinantes do requerimento de colateral em operações de crédito de curto prazo, pactuadas com empresários formais no Brasil, utilizam dois modelos de regressão. O primeiro, baseado em uma variável dependente limitada, é estimado por regressão Logit para dados em painel. O segundo, considerando o logaritmo natural da variável dependente percentual de colateral vinculado a empréstimos bancários empresariais $\left(\right.$ (Follateral $_{j t}$ ), é estimado por GMM para dados em painel.

\footnotetext{
${ }^{4}$ Um entendimento formal dos mencionados testes pode ser obtido em Sargan (1958) e Roodman (2007).
} 
Observa-se, ainda, que o primeiro modelo de regressão testa as quatro primeiras hipóteses, (H1) a (H4b), enquanto que o segundo testa somente a quinta hipótese, (H5).

\subsubsection{Modelo Logit para Dados em Painel}

Preliminarmente à estimativa com dados em painel, aplicou-se o teste de Hausman (1978) para selecionar entre modelos de efeitos fixos ou aleatórios. O valor da estatística calculada, próximo de 950, permite rejeitar a hipótese nula de ausência de correlação entre heterogeneidade individual e regressores nos modelos estimados. Isto indica que o modelo de efeitos fixos deve ser utilizado nas estimativas com dados de painel. Além disso, este resultado é um indicativo de que a hipótese de que efeitos fixos de devedores explicam a utilização de colateral em empréstimos bancários de curto prazo não pode ser rejeitada.

A Tabela 1 apresenta os resultados empíricos obtidos com o modelo Logit para dados em painel, os quais subsidiam as análises feitas a seguir, referentes às quatro primeiras hipóteses apresentadas na subseção 2.1. Observe que os efeitos cruzados, chamados EC na tabela, foram modelados pela interação entre variáveis dummies e regressores para testar hipóteses específicas.

Tabela 1: Determinantes de Colateral: Economia Empresarial

\begin{tabular}{|c|c|c|c|c|}
\hline \multirow{2}{*}{ Variável } & \multicolumn{2}{|c|}{ Logit sem EC } & \multicolumn{2}{|c|}{ Logit com EC } \\
\hline & Coeficiente & P-value & Coeficiente & P-value \\
\hline Defaultt-1 & -0.352521 & 0.281 & 2.094 .595 & 0.046 \\
\hline Defaultt+1 & -0.882836 & 0.000 & -0.890692 & 0.000 \\
\hline Herfindahl & -0.526612 & 0.000 & -0.432145 & 0.000 \\
\hline Ln(Duration) & 0.054809 & 0.405 & 0.324759 & 0.328 \\
\hline Geographicrisk & -0.120512 & 0.307 & -0.123403 & 0.295 \\
\hline BA*Unemployment & 0.065352 & 0.068 & 0.066419 & 0.064 \\
\hline MG*Unemployment & -0.124139 & 0.000 & -0.123541 & 0.000 \\
\hline PE*Unemployment & -0.081254 & 0.116 & -0.080235 & 0.121 \\
\hline RJ'Unemployment & 0.225929 & 0.000 & 0.227142 & 0.000 \\
\hline RS*Unemployment & -0.162912 & 0.000 & -0.162863 & 0.000 \\
\hline SP*Unemployment & -0.058820 & 0.017 & -0.057040 & 0.021 \\
\hline Segment-commerce & 0.457478 & 0.341 & 0.458735 & 0.341 \\
\hline Segment-industry & 0.695814 & 0.273 & 0.697643 & 0.272 \\
\hline Ageentrepreneur2 & 0.107002 & 0.063 & 0.103749 & 0.072 \\
\hline Ageentrepreneur10to20 & -0.005551 & 0.555 & -0.004935 & 0.600 \\
\hline Ageentrepreneur20 & 0.004685 & 0.764 & 0.006131 & 0.696 \\
\hline UseEletronic & 0.451253 & 0.008 & 0.472931 & 0.016 \\
\hline Ln(Debt) & -0.008359 & 0.368 & -0.007920 & 0.394 \\
\hline Real interest rate & 0.032058 & 0.000 & 0.028660 & 0.000 \\
\hline Ln(Contractvalue) & 0.982308 & 0.000 & 0.982712 & 0.000 \\
\hline UseEletronic* $\ln$ (Duration) & & & -0.014109 & 0.815 \\
\hline Herfindahl* $\ln$ (Duration) & & & -0.046946 & 0.249 \\
\hline \multirow[t]{2}{*}{ Defaultt- $1^{*} \ln$ (Duration) } & & & -1.680 .355 & 0.014 \\
\hline & \multicolumn{2}{|c|}{ Logit sem EC } & \multicolumn{2}{|c|}{ Logit com EC } \\
\hline Log likelihood & \multicolumn{2}{|c|}{$-23.147 .862$} & \multicolumn{2}{|c|}{-23144.12} \\
\hline$\chi_{2}$ variables & \multicolumn{2}{|c|}{11274.51} & \multicolumn{2}{|c|}{11281.99} \\
\hline Pseudo $R^{2}$ & \multicolumn{2}{|c|}{0.1958} & \multicolumn{2}{|c|}{0.196} \\
\hline
\end{tabular}

Nota: estimativa logit e dados de painel com 75.832 observações. EC representa efeitos cruzados.

O coeficiente estimado da variável $\operatorname{Default}_{(j t-1)}$, que testa (H1), foi estatisticamente significante ao nível de $5 \%$ se significância. Isso não permite rejeitar a hipótese de que devedores com menor qualidade de crédito são mais propensos a vincular colateral em empréstimos bancários de curto prazo do que aqueles com maior qualidade de crédito, no caso do segmento empresarial brasileiro. 
Por outro lado, o sinal negativo e estatisticamente significante a $1 \%$ de $\operatorname{Default}_{(j t+1)}$ mostra que a probabilidade de vinculação de colateral é maior entre aqueles devedores que cumprem suas promessas financeiras em $t+1 .^{5}$

Este fato indica que o efeito dominante na amostra analisada refere-se ao grupo que detém informação privada no momento da contratação do empréstimo bancário e, revela uma possível ocorrência do problema de seleção adversa no relacionamento de empréstimos credor-devedor na economia empresarial sob estudo.

Entretanto, o sinal positivo e estatisticamente significante ao nível de $10 \%$ do coeficiente da variável Ageentrepreneur2 indica que aquela informação assimétrica pode não se verificar para empresários com menor experiência empresarial. Ou ainda, estes empresários são mais prováveis de vincular colateral em operações de empréstimos para sinalizar sua qualidade de crédito. Adicionalmente, os coeficientes das variáveis Ageentrepreneur10to20, firmas individuais com idade entre 10 e 20 anos, e Ageentrepreneur20, firmas individuais com idade acima de 20 anos, não se mostraram estatisticamente significantes.

A terceira hipótese é testada pelos valores estimados dos coeficientes da variável Duration $_{(j t-1)}$, $\mathrm{b}_{3}$, e do efeito cruzado desta variável com Default $(j t-1), \mathrm{b}_{4}$. Se Default $(j t-1)=1$ estatisticamente, então, tem-se que a soma daqueles coeficientes é $\mathrm{b}_{3}+\mathrm{b}_{4}=-1.68$, estatisticamente significante a $10 \%$ (conforme revela o valor $-p$ de 0.0742 do teste de Wald). Tal resultado corrobora a hipótese de Boot e Thakor (1994), segundo a qual um maior tempo de relacionamento comercial devedor-credor pode contribuir para construção de confiança e redução do risco moral. Além disso, indica uma menor probabilidade de requerimento de colateral pelo credor para todos os devedores durante o período analisado.

Note que o coeficiente negativo e estaticamente significante a $1 \%$ da variável Herfindahl $l_{t}$ não permite rejeitar a hipótese (H4a). Então, considerando que competição e rivalidade são maiores em mercados competitivos, esta evidência empírica sugere a validade da hipótese de que competição eleva a probabilidade de utilização de colateral em empréstimos de curto prazo concedidos a empresários formais no Brasil. Adicionalmente, observe que o coeficiente referente ao efeito cruzado entre as variáveis $\operatorname{Duration}_{(j t-1)}$ e Herfindahl, , que testa (H4b), não se mostrou estatisticamente significante. Isso indica que concentração em mercados de crédito não é afetada por efeitos de tempo provenientes do relacionamento devedor-credor sobre a probabilidade de vinculação de colateral na economia empresarial sob referência.

Note também que o valor positivo do coeficiente da variável UseEletronic ${ }_{j t}$, estatisticamente significante a 95\% de confiança, indica a existência de uma relação direta entre a probabilidade de requerimento de colateral e a utilização de meio eletrônico para realização de transações bancárias. Uma possível interpretação desse resultado empírico pode estar associada ao fato de que se bancos decidem reduzir custos de transação para incentivar clientes a utilizarem canais eletrônicos para efetuar operações bancárias teriam, por consequência, uma redução em suas margens de ganho unitárias. Logo, os bancos tenderiam a proteger suas margens comerciais requerendo uma maior utilização de colateral em operações de crédito formalizadas em meio eletrônico. Entretanto, o coeficiente da variável com efeito cruzado UseEletronic ${ }_{j t}{ }^{*} \ln$ (duration) não se mostrou estatisticamente significante, indicando que a

\footnotetext{
${ }^{5}$ Note que o cumprimento da promessa de pagamento de um empréstimo comercial poderia ser motivado por outros fatores, dentre eles destacando-se:

i o colateral vinculado pode ter maior valor de mercado para o mutuário do que para o banco, no momento do vencimento da operação de crédito;

ii a pequena empresa pode ter interesse de manter um histórico de elevada qualidade de crédito no sistema bancário, considerando que esta pode ser sua única fonte de acesso a crédito;
}

iii a decisão de pagamento pode ser uma condição de maximização de lucro para a firma. 
probabilidade de vinculação de colateral devido ao uso de meio eletrônico não é afetada pelo tempo de relacionamento com o tomador.

Note ainda que os coeficientes das variáveis relacionadas com o setor de atuação do empresário, Segment-commerce $_{(j t-1)}$ e Segment-industry $(j t-1)$, existência de dívidas, $\ln \left(\operatorname{Debt}_{(j t-1)}\right)$, e risco geográfico, Geographic risk $(t-1)$, não se mostraram estatisticamente significantes. Este resultado evidencia, de certa forma, uma relativa irrelevância destas variáveis setoriais na explicação do uso de colateral em empréstimos comerciais, ou que esta vinculação seria mais bem explicada por aqueles efeitos subjacentes às hipóteses descritas acima, para o caso brasileiro. Então, estas evidências empíricas, em conjunto com as evidências observadas na avaliação da hipótese (H2) acima, sugerem a corroboração de resultados da literatura, no sentido de que determinantes de colateral em empréstimos bancários comerciais podem ser distintos entre diferentes mercados de crédito e entre devedores com diferentes níveis de experiência. ${ }^{6}$

Além disso, note que coeficientes de variáveis utilizadas como controles, quando estatisticamente significantes, apresentaram sinais distintos entre diferentes unidades da federação brasileira. Isso ocorreu com a variável "desemprego" nas regiões metropolitanas de BA, MG, PE, RJ, RS e SP. Dependendo da unidade de federação, as evidências indicam uma maior ou menor probabilidade de vinculação de colateral se comparada com as demais unidades da federação pertencentes ao grupo de controle utilizado para esta variável. Então, tais resultados empíricos revelam que efeitos regionais podem impactar de forma distinta os requerimentos de colateral na economia empresarial brasileira no mencionado período.

4.2.1.1. Efeitos Marginais A Tabela 2 apresenta efeitos marginais e semi-elasticidades sobre a probabilidade de requerimentos de colateral na economia empresarial brasileira, usando o modelo que incorpora efeitos cruzados. Vale lembrar que, nesse modelo, o efeito marginal contempla o efeito total dos coeficientes da variável, correspondente à soma do efeito individual com o efeito cruzado.

Tabela 2: EstimaçãoLogit: Efeitos Marginais

\begin{tabular}{|c|c|c|}
\hline Variável & Efeitos Marginais & Semi-Elasticidade \\
\hline Default $_{t-1}$ & $0.41^{*}$ & $0.24 \%$ \\
\hline Default $_{t+1}$ & $-0.89^{* *}$ & $-0.67 \%$ \\
\hline Herfindahl & $-0.05^{* *}$ & $-63.32 \%$ \\
\hline Ln(Duration) & -0.09 & $-7.77 \%$ \\
\hline Geographicrisk & -0.13 & $-0.14 \%$ \\
\hline BA*Unemployment & 0.11 & $8.86 \%$ \\
\hline MG*Unemployment & $-0.25^{* *}$ & $-26.78 \%$ \\
\hline PE*Unemployment & -0.10 & $-3.53 \%$ \\
\hline RJ*Unemployment & $0.27^{* *}$ & $6.25 \%$ \\
\hline RS*Unemployment & $-0.28^{* *}$ & $-20.31 \%$ \\
\hline SP*Unemployment & $-0.16^{*}$ & $-33.36 \%$ \\
\hline Segment-commerce & 0.45 & $3.47 \%$ \\
\hline Segment-industry & 0.69 & $0.08 \%$ \\
\hline Ageentrepreneur2 & 0.10 & $0.28 \%$ \\
\hline Ageentrepreneur10to20 & -0.01 & $-0.08 \%$ \\
\hline Ageentrepreneur20 & 0.01 & $0.03 \%$ \\
\hline UseEletronic & $0.47^{*}$ & $28.75 \%$ \\
\hline Ln(Debt) & -0.01 & $-1.01 \%$ \\
\hline Real interest rate & $0.03^{* *}$ & $2.82 \%$ \\
\hline Ln(Contractvalue) & $0.01^{* *}$ & $1.03 \%$ \\
\hline
\end{tabular}

\footnotetext{
${ }^{6}$ Em razão de que alguns coeficientes de variáveis de controle não se mostraram estatisticamente significantes a 5\%, decidiu-se proceder a uma análise de sensibilidade por meio de outra regressão logit com dados em painel, no qual aqueles controles foram removidos das estimativas. Os resultados dos testes das hipóteses (H1) a (H4b), porém, mantiveram-se inalterados.
} 
Por exemplo, considerando que todas as outras variáveis se mantenham constantes, é observado que firmas individuais que apresentam um histórico de default anterior ao momento da concessão do crédito impactam positivamente a probabilidade de requerimento de colateral em 0.41 , com nível de confiança estatística de $95 \%$. Diferentemente, para empresários que não apresentam aquele histórico e com base no efeito marginal de Default $_{(j t+1)}$, estatisticamente significante a $1 \%$, é observado que o impacto na mencionada probabilidade é negativo, igual a -0.89 . Esse resultado, possivelmente, é resultante da mencionada evidência de efeito dominante do grupo que detém informação privada antes da contratação do empréstimo bancário.

Quanto à avaliação de semi-elasticidades, observa-se que uma elevação de $1 \%$ na taxa de desemprego em regiões metropolitanas provoca diferentes efeitos regionais. Para a região metropolitana do Rio de Janeiro, por exemplo, há uma elevação média de 6\% na probabilidade de vinculação de colateral. Já na região metropolitana de São Paulo, $S P^{*} U_{n e m p l o y m e n t}$, os resultados empíricos sugerem uma redução naquela probabilidade em torno de $30 \% \mathrm{em}$ empréstimos empresariais.

\subsubsection{Modelo GMM para Dados em Painel}

A Tabela 3 apresenta os resultados obtidos na avaliação da quinta hipótese, (H5). Observe-se que neste teste foram utilizadas as mesmas variáveis explicativas usadas na estimativa logit com dados em painel descrita na seção 4.2.1.

Tabela 3: Requerimento de Colateral: Fator Contínuo

\begin{tabular}{lcccc}
\hline \multirow{2}{*}{ Variável } & \multicolumn{2}{c}{ Painel sem EC } & \multicolumn{2}{c}{ Painel com EC } \\
\cline { 2 - 5 } & Coeficiente & P-value & Coeficiente & P-value \\
\hline Constant & 1.024 .706 & 0.000 & 0.996359 & 0.000 \\
Defaultt-1 & 0.017207 & 0.517 & 0.200617 & 0.117 \\
Defaultt+1 & -0.064179 & 0.000 & -0.062294 & 0.000 \\
Herfindahl & -0.105902 & 0.000 & -0.105014 & 0.000 \\
Ln(Duration) & 0.001509 & 0.230 & 0.011460 & 0.380 \\
Geographicrisk & -0.019037 & 0.004 & -0.018807 & 0.004 \\
BA*Unemployment & 0.000257 & 0.279 & 0.000280 & 0.236 \\
MG*Unemployment & -0.001315 & 0.000 & -0.001314 & 0.000 \\
PE*Unemployment & 0.002332 & 0.000 & 0.002329 & 0.000 \\
RJ*Unemployment & 0.001922 & 0.000 & 0.001924 & 0.000 \\
RS*Unemployment & -0.002779 & 0.000 & -0.002766 & 0.000 \\
SP*Unemployment & 0.000862 & 0.000 & 0.000852 & 0.000 \\
Segment-commerce & 0.045713 & 0.000 & 0.045625 & 0.000 \\
Segment-industry & 0.054805 & 0.000 & 0.054470 & 0.000 \\
Ageentrepreneur2 & 0.040673 & 0.000 & 0.040896 & 0.000 \\
Ageentrepreneur10to20 & -0.002473 & 0.000 & -0.002415 & 0.000 \\
Ageentrepreneur20 & -0.001222 & 0.000 & -0.001145 & 0.000 \\
UseEletronic & 0.051387 & 0.000 & 0.070294 & 0.000 \\
Ln(Debt) & 0.054738 & 0.000 & 0.053341 & 0.000 \\
Real interest rate & 0.005852 & 0.000 & 0.005904 & 0.000 \\
Ln(Contractvalue) & -0.056257 & 0.000 & -0.053948 & 0.000 \\
UseEletronic*ln(Duration) & & & -0.011592 & 0.000 \\
Herfindahl*ln(Duration) & & & -0.000352 & 0.800 \\
Defaultt-1*ln(Duration) & & & -0.124487 & 0.102 \\
\hline Estimação GMM & Sargan Test & Difference-Sargan Test \\
& 0.956 & 0.3280 & 1.363 & 0.2430 \\
\hline Panel sem EC & 0.799 & 0.3714 & 1.134 & 0.2870 \\
Painel com EC & & & & \\
Estimativa GMM para dados em painel, com 172.836 observações. EC representa efeitos cruzados. \\
\end{tabular}

Novamente, preliminarmente a estimação GMM com dados em painel, aplicou-se o teste de Hausman (1978) para selecionar entre os modelos de efeitos fixos ou aleatórios. A estatística calculada permite rejeitar a hipótese nula de ausência de correlação entre heterogeneidade individual e regressores no modelo estimado, indicando que se deve proceder à estimação do modelo de efeitos fixos. 
Na Tabela 3, observa-se que as estatísticas do teste de Sargan (1958) não permitem rejeitar a hipótese nula de sobreidentificação nas estimativas de GMM com dados em painel. ${ }^{7}$ Adicionalmente, as estatísticas calculadas por meio da aplicação do teste difference-Sargan em ambas estimativas não permitem rejeitar a hipótese de especificação correta do modelo utilizado.

De acordo com os resultados reportados na Tabela 3, não se pode rejeitar a validade da quinta hipótese, (H5), o que corrobora outras evidências da literatura a seguir exemplificada. Conforme sugerido por Boot et alii (1991), em economias com elevada taxa básica de juros, um banco, visando proteger margens comerciais e minimizar efeitos de problemas de moral hazard, escolhe exigir um elevado valor de colateral em operações de empréstimos bancários ao invés de acrescentar aquele efeito incremental na taxa de juros do contrato de crédito. Alternativamente, naquelas condições, pode-se interpretar que a aplicação de um prêmio de risco adicional poderia contribuir para agravar o problema de risco moral, assim como, para uma possível queda na qualidade das demandas por empréstimos, o que motivaria a decisão do credor de requerer um maior valor de colateral.

Os resultados empíricos constantes da terceira e quarta colunas da Tabela 3 também revelam que foram mantidos os sinais dos coeficientes das variáveis utilizadas para testar aquelas quatro primeiras hipóteses, conforme reportado na Tabela 2. Entretanto, isto não se verifica para coeficientes de alguns controles que mudam de sinal ou se tornam estatisticamente significantes possivelmente devido ao método de estimação utilizado, tal como observado previamente por Jiménez et alii (2006).

\section{CONCLUSÃO}

O objetivo deste trabalho foi avaliar empiricamente os determinantes de vinculação de colateral em empréstimos bancários em uma economia empresarial, no horizonte de tempo de curto prazo, seguindo os modelos de Jiménez et alii (2006) e Steijvers e Voordeckers (2009). Para tanto, foi utilizada uma amostra de aproximadamente 170 mil empréstimos comerciais provenientes de um segmento específico do mercado de crédito brasileiro. Foram conduzidas estimações logit e GMM para dados em painel no período de 2004 a 2008.

Preliminarmente, a base de dados disponível, a qual é composta de informações de pequenos e médios empresários, não permitiu detalhar questões relacionadas com a natureza do colateral, se interno ou externo, nem informações relevantes de empresários, tais como, variáveis econômico-financeiras. De fato, a ausência de informações limita o escopo dos nossos testes. Entretanto, pondera-se que as evidências empíricas obtidas não foram afetadas devido a inclusão de variáveis que controlam para esses efeitos não-observáveis, incluindo efeitos fixos.

Os resultados empíricos sugerem a existência de fatores que impactam os requerimentos de colateral em operações de crédito formalizadas com empresários formais no Brasil. Destacam-se efeitos de experiência empresarial, taxa de juros da economia, desemprego regional e competição no mercado de crédito brasileiro. Esses resultados também corroboram evidências anteriores de Jiménez et alii (2006) no sentido de que determinantes de colateral em empréstimos comerciais podem ser distintos entre diferentes mercados de crédito e entre devedores com diferentes níveis de experiência. Adicionalmente, os resultados revelam que aqueles requerimentos podem ser distintos entre diferentes unidades da federação brasileira, no período proposto para análise.

Note ainda que os testes empíricos estimados nesta pesquisa possivelmente não contemplam em sua totalidade os vários componentes que possivelmente explicam o uso de colateral em empréstimos bancários comerciais, tais como:

i sua disponibilidade e distribuição entre agentes econômicos,

\footnotetext{
${ }^{7}$ Foram utilizadas duas defasagens e uma defasagem das variáveis explicativas Contract value ${ }_{j t}$ e Real interest rate $_{t}$, respectivamente, como instrumentos.
} 
ii sua liquidez e valor diferenciado entre as partes,

iii efeitos macroeconômicos,

iv os problemas de incentivos que afetam tanto tomadores como emprestadores.

Logo, reconhecemos que há mais elementos a serem analisados em pesquisas futuras, as quais também poderiam focar em dados de contratos de crédito de longo prazo e em outros segmentos do mercado de crédito brasileiro. Além disso, devem-se utilizar bases de dados mais amplas, assim como analisar os impactos provenientes do requerimento de capital regulatório para bancos no contexto do Acordo de Basiléia II.

\section{BIBLIOGRAFIA}

Berger, A. N., Espinosa-Vega, M. A., Frame, W. S., \& Miller, N. H. (2011). Why do Borrowers Pledge Collateral? New Empirical Evidence on the Role of Asymmetric Information. Journal of Financial Intermediation, 20:55-70.

Bester, H. (1985). Screening vs. Rationing in Credit Markets with Imperfect Information. American Economic Review, 75:850-855.

Boot, A. W. \& Thakor, A. V. (1994). Moral Hazard and Secured Lending in an Infinitely Repeated Credit Market Game. International Economic Review, 35:899-920.

Boot, A. W. \& Thakor, A. V. (2000). Can Relationship Banking Survive Competition? Journal of Finance, 55:679-713.

Boot, A. W. A., Thakor, A. V., \& Udell, G. F. (1991). Secured Lending and Default Risk: Equilibrium Analysis, Policy Implications and Empirical Results. The Economic Journal, 101:458-472.

Chan, Y. \& Kanatas, G. (1985). Asymmetric Valuations and the Role of Collateral in Loan Agreements. Journal of Money, Credit and Banking, 17:84-95.

De Araújo, L. A. D. \& Jorge-Neto, P. M. (2007). Risco e Competição Bancária no Brasil. Revista Brasileira de Economia, 61:175-200.

Farinha, L. A. \& Santos, J. A. (2002). Switching from Single to Multiple Bank Lending Relationships: Determinants and Implications. Journal of Financial Intermediation, 11:124-151.

Greenbaum, S. I., Kanatas, G., \& Venezia, I. (1989). Equilibrium Loan Pricing Under the Bank-Client Relationship. Journal of Banking and Finance, 13:221-235.

Greene, W. H. (1997). Econometric Analysis. Technical report, Prentice-Hall, New York.

Hamilton, J. (1994). Time Series Analysis. Technical report, Princeton University Press, New Jersey.

Hansen, L. P. (1982). Large Sample Properties of Generalized Method of Moments. Estimators. Econometrica, 50:1029-1054.

Hausman, J. (1978). Specification Tests in Econometrics. Econometrica, 46:1251-1272.

Hayashi, F. (2000). Econometrics. Technical report, Princeton University Press, New Jersey.

Hsiao, C. (2006). Panel Data Analysis - Advantages and Challenges. 
Inderest, R. \& Mueller, H. (2007). A Lender-Based Theory of Collateral. Journal of Financial Economics, 84:826-859.

Jiménez, G., Salas, V., \& Saurina, J. (2006). Determinants of Collateral. Journal of Financial Economics, $81: 255-281$.

Jiménez, G., Salas, V., \& Saurina, J. (2009). Organizational Distance and Use of Collateral for Business Loans. Journal of Banking and Finance, 33:234-243.

Leland, H. E. \& Pyle, D. H. (1977). Informational Asymmetries, Financial Structure, and Financial Intermediation. Journal of Finance, 32:371-387.

Nakane, M. I. (2002). Test of Competition in Brazilian Banking. Estudos Econômicos, 32:203-224.

Ono, A. \& Uesugi, I. (2009). The Role of Collateral and Personal Guarantees in Relationship Lending: Evidence from Japan's Small Business Loan Market. Journal of Money, Credit \& Banking, 41:935-960.

Ozawa, C. Y. \& Nakane, M. I. (2009). Taxas de Juros, Garantias e Tempo de Relacionamento nos Contratos de Crédito: Evidências para o Brasil.

Petersen, M. A. \& Rajan, R. G. (1994). The Benefits of Lending Relationships: Evidence from Small Business Data. Journal of Finance, 49:3-37.

Petersen, M. A. \& Rajan, R. G. (1995). The Effect of Credit Market Competition on Lending Relationships. Quarterly Journal of Economics, 110:407-444.

Roodman, D. (2007). A Short Note on the Theme of Too Many Instruments.

Sargan, J. D. (1958). The Estimation of Economic Relationships using Instrumental Variables. Econometrica, 26:393-415.

Steijvers, T. \& Voordeckers, W. (2009). Collateral and Credit Rationing: A Review of Recent Empirical Studies as a Guide for Future Research. Journal of Economic Surveys, 23:924-946.

Voordeckers, W. \& Steijvers, T. (2006). Business Collateral and Personal Commitments in SME Lending. Journal of Banking and Finance, 30:3067-3086.

Wooldridge, J. M. (2001). Econometric Analysis of Cross Section and Panel Data. Technical report, The MIT Press, Cambridge. 


\section{A. APÊNDICE A - REGRESSOR ENDÓGENO}

De acordo com Hayashi (2000), um regressor é dito endógeno se não é ortogonal ao termo de erro, isto é, $E\left[X_{j t k}^{\prime} . e_{j t}\right] \neq 0$ para algum regressor $k$. Neste contexto, por simplicidade, admitindo que todos os demais coeficientes constantes do modelo acima sejam estatisticamente não-significantes, exceto o coeficiente do regressor ContractValue ${ }_{j t} \in X_{j t k}^{\prime}$, pode-se escrever:

$$
E\left[\text { ContractValue }_{j t .} e_{j t}\right]=E\left[\text { ContractValue }_{j t} .\left(\text { Fcollateral }_{j t}-b_{7} \text { ContractValue }_{j t}\right)\right]
$$

Considerando $e_{j} t=\left(\right.$ Fcollateral $_{j t}-b_{7}$ ContractValue $\left._{j t}\right)$. Então, substituindo Fcollateral $_{j t}=(1+$ CollateralValue $_{j t}$ /ContractValue ${ }_{j t}$ ) e efetuando a multiplicação indicada no lado direito nesta última expressão, resulta:

$$
\begin{gathered}
E\left[\text { ContractValue }_{j t .} e_{j t}\right]= \\
E\left[\text { ContractValue }_{j t}+\text { CollateralValue }_{j t}+\text { ContractValue }_{j t} \cdot\left(-b_{7} \text { ContractValue }_{j t}\right)\right]>0
\end{gathered}
$$

pois, por (H5) é esperado $b_{7}<0$. Com efeito, se a condição de ortogonalidade não é satisfeita para esta variável explicativa, pela mencionada definição, tem-se que $X_{j t k}^{\prime}$ é um regressor endógeno. Essa possibilidade será avaliada empiricamente para a amostra testada por meio do teste de Hausman (1978).

Logo, a estimativa do modelo econométrico proposto para teste de (H5), conforme equação (2) anterior, requer o uso de variáveis instrumentais. Neste caso, sugere-se a utilização do método dos momentos generalizados, GMM, cujo estimador IV é um caso particular.

\section{B. APÊNDICE B - ESTIMAÇÃO LOGIT COM DADOS EM PAINEL}

Seguindo Greene (1997), para estimação dos modelos lineares com dados em painel, em uma representação geral, pode-se utilizar a seguinte equação:

$$
y_{j t}=x_{j t}^{\prime} \beta+\eta_{j}+u_{j t}
$$

em que $y_{j t}$ representa a variável dependente do modelo, $x_{j t}^{\prime}$, representa um vetor das variáveis explicativas, $\beta$ representa o vetor de parâmetros, $\eta_{j}$ representa choques invariantes ao longo do tempo, específicos a cada firma individual $\mathrm{j}$, e $u_{j t}$ representa choques aleatórios dependentes do tempo.

Para o caso de modelos não-lineares, seguindo Wooldridge (2001) e Hsiao (2006), pode-se utilizar o seguinte modelo logit com dados de painel:

$$
\operatorname{Prob}\left(y_{j t}=1 \mid x_{j t}, \alpha_{j}\right)=F(z)=\frac{e^{z}}{1+e^{z}}=\frac{e^{\left(\beta x_{j t}+\alpha_{j}\right)}}{1+e^{\left(\beta x_{j t}+\alpha_{j}\right)}}
$$

onde $y_{j t}$ representa uma variável dependente limitada, $F(z)$ é uma função logística que assume valores entre zero e um, isto é, $0<F(z)<1$ para todos os números reais, indicando que as probabilidades de resposta da estimativa ficam estritamente neste intervalo, e $z$ é um vetor de variáveis explicativas descritas anteriormente. 